IdeAs

Idées d'Amériques

$8 \mid 2016$

Ressources minières dans les Amériques: Mutations d'un continent

\title{
De Trump candidat à Trump président : un mandat de gouvernement ambigu
}

Vincent Michelot

\section{(2) OpenEdition \\ Journals}

Édition électronique

URL : https://journals.openedition.org/ideas/1694

DOI : $10.4000 /$ ideas. 1694

ISSN : 1950-5701

Éditeur

Institut des Amériques

Référence électronique

Vincent Michelot, " De Trump candidat à Trump président : un mandat de gouvernement ambigu », IdeAs [En ligne], 8 | 2016, mis en ligne le 16 décembre 2016, consulté le 18 octobre 2022. URL : http:// journals.openedition.org/ideas/1694; DOI : https://doi.org/10.4000/ideas.1694

Ce document a été généré automatiquement le 18 octobre 2022.

\section{(c) $(1)(9)$}

Creative Commons - Attribution - Pas d'Utilisation Commerciale - Pas de Modification 4.0 International - CC BY-NC-ND 4.0

https://creativecommons.org/licenses/by-nc-nd/4.0/ 


\title{
De Trump candidat à Trump président : un mandat de gouvernement ambigu
}

\author{
Vincent Michelot
}

1 Si l'on arrête aux résultats bruts du 9 novembre 2016, le triomphe de Donald Trump est total : il l'emporte avec une marge confortable de 74 grands électeurs dans le collège électoral, le seul résultat qui compte, ce malgré un écart très large $(2,7 \mathrm{M}$ de voix à ce jour) en faveur de Hillary Clinton dans les suffrages populaires à l'échelle nationale; le Parti républicain, bien que très menacé par le simple fait qu'il devait " défendre » 24 sièges au Sénat sur les 34 en jeu, conserve sa majorité à la Chambre haute, ce probablement jusqu'en 2020 au moins, puisque lors des élections de mi-mandat de 2018 ce seront 25 sortants démocrates sur un total de 33 sièges qui devront à leur tour «défendre » leur poste ; de la même manière, à la Chambre des représentants, les Démocrates ne gagnent que 6 sièges, ce qui est largement insuffisant pour retrouver la majorité puisqu'ils n'auront à la rentrée parlementaire en janvier 2017 que 194 sièges face au 241 des Républicains. Là encore, ils ne peuvent espérer rapidement retrouver le poste de speaker tant le nombre de circonscriptions compétitives est faible, ce qui met les Républicains à l'abri, même dans le cas d'un fort mouvement de mécontentement anti-Trump en 2018. Trente quatre gouverneurs sur les cinquante sont républicains et, dans 32 États le Parti de l'éléphant dispose de la majorité dans les deux chambres de l'assemblée législative. Il est donc statistiquement très fortement probable que Donald Trump réalise l'ensemble de son (premier) mandat avec une majorité républicaine au Congrès et dans les États, voire que cette majorité s'amplifie et se consolide, à court terme d'abord en 2018, à moyen ou long terme par la suite avec les conséquences mécaniques du découpage électoral qui fera suite au recensement de 2020 et sera majoritairement opéré par des assemblées législatives à majorité républicaine.

Cerise sur le gâteau électoral du futur 45 ème président des États-Unis, Donald Trump va trouver dans sa nouvelle boîte à outils la possibilité de nommer dès ses premières semaines à la Maison Blanche un juge à la Cour suprême des États-Unis qui va faire pencher la balance idéologique du haut tribunal américain en faveur des conservateurs. 
Un siège est en effet vacant depuis le décès en février 2016 d'Antonin Scalia et la manœuvre d'obstruction réussie du leader républicain à la Chambre haute, Mitch McConnell, qui a bloqué l'examen par la Commission judiciaire de la proposition de nomination faite par le président Obama. C'était un pari osé puisque une victoire de Hillary Clinton, qui était statistiquement très probable jusqu'à la veille du scrutin, lui aurait donné la possibilité de nommer un juge nettement plus progressiste que Merrick Garland, le magistrat très expérimenté mais aussi très modéré de la Cour d'appel fédérale du District de Columbia sur lequel s'était porté le choix du président. Donald Trump aura peut-être même la possibilité d'ajouter une deuxième, voir une troisième nomination à son palmarès, avec un fort pouvoir idéologique transformateur puisque les deux magistrats les plus âgés sont la plus progressiste, Ruth Bader Ginsburg, et un modéré centriste qui a souvent fait basculer la jurisprudence de la Cour contre les conservateurs.

2 Enfin, un certain nombre d'indices pointent dans la direction d'un mandat de gouvernement confié à Donald Trump qui serait beaucoup plus positif que celui de la candidate démocrate si elle l'avait emporté. L'essentiel du discours de campagne de Hillary Clinton dans les dernières semaines consistait en effet à présenter Donald Trump comme inapte à l'exercice de la fonction exécutive et comme dangereux pour la démocratie américaine. Le programme de la Démocrate est donc passé au deuxième plan. De son côté le milliardaire new-yorkais, tout en lançant des attaques personnelles très dures contre Hillary Clinton, est parvenu à s'incarner en tant qu'agent du changement politique porteur de propositions fortes dotées d'un énorme pouvoir symbolique. Il va de plus bien au-delà d'une alternance partisane puisque, en gagnant en large mesure contre son propre parti, il se présente aussi comme un agent de transformation et de refondation du Parti républicain ; par ailleurs, en violant délibérément et joyeusement tous les codes du politique américain, c'est tout Washington qu'il semble prêt à rénover et à bouleverser. De ce fait, tout son programme est dessiné, dans sa radicalité (interdire aux Musulmans d'entrer sur le territoire des États-Unis, construire un mur à la frontière avec le Mexique et le faire payer à son voisin et partenaire dans l'ALENA, déporter plusieurs millions d'immigrants en situation irrégulière, recourir à la torture dans la lutte contre le terrorisme, ouvrir la boîte de Pandore d'une "nucléarisation » du Japon ou de la Corée du Sud...) et sa violence verbale pour incarner le changement et, par ricochet, faire oublier la personnalité et l'inexpérience de l'agent de ce changement. Donald Trump a donc bien été élu pour mettre en œuvre ses idées et la forte participation des Blancs non diplômés de l'enseignement supérieur (qui contraste avec une mobilisation médiocre des groupes traditionnellement importants dans les coalitions électorales démocrates), un groupe qui s'était d'abord détaché du Parti de l'âne, puis rapproché fortement des Républicains et avait enfin, à partir de 1992, éprouvé un sentiment de déception voire de trahison vis-à-vis du Parti de l'éléphant, en est un symbole fort. Des électeurs qui s'étaient petit à petit éloignés du processus électoral sont revenus en masse d'abord s'inscrire sur les listes électorales, puis voter. Ce serait une erreur que de considérer ce vote comme simplement protestataire ou ressortant du rejet de la personne de Hillary Clinton et des idées et politiques publiques qu'elle incarnait. Clairement, une majorité d'Américains qui se sont exprimés le 8 novembre souhaitent un contrôle renforcé de l'immigration, une redéfinition de la mondialisation dans ses dimensions économiques et culturelles, plus de protection de l'État et un regard 
nouveau sur la place et les responsabilités des États-Unis dans la communauté des nations.

Donald Trump se trouve donc dans une position de force que jamais le Parti républicain n'a connue au 20ème ou au 21ème siècle. Il contrôle avec son parti tous les leviers du pouvoir, au fédéral et au fédéré. Pourtant, les éléments de fragilité et d'incertitude sont extrêmement nombreux et laissent à penser que cette toute-puissance n'est que virtuelle. C'est donc uniquement dans un contexte très particulier qu'elle pourra devenir réelle et exprimer son potentiel transformateur.

En effet, si Donald Trump a cassé les codes de campagne et réécrit le guide du candidat à la présidence, il ne s'est pas pour autant affranchi de toutes les contraintes institutionnelles et idéologiques du système politique américain. On commencera par rappeler que la présidence des États-Unis n'est ni omnipotente, ni dégagée des contrepouvoirs, ni insensible ou inaccessible aux mécanismes économiques et sécuritaires internationaux, quand bien même chef de l'exécutif et majorité au Congrès sont de la même couleur partisane. Le président élu a fait toute sa campagne sur la base d'un slogan qui souligne l'extrême solitude du futur occupant du Bureau ovale : «I alone can fix it! ». Cet exercice solitaire du pouvoir est lui-même fondé sur une analogie qui fleure bon les années 1920 lorsque l'on comparait la gestion des États-Unis à celle d'une grande entreprise comme General Motors et donc la fonction présidentielle à celle d'un capitaine d'industrie. Cette analogie est aujourd'hui délicieusement surannée ou dangereusement dysfonctionnelle, tout comme peut l'être la comparaison entre une négociation commerciale entre deux entreprises (ou entre un client et un prestataire de services) et la négociation d'un traité de libre échange, d'un accord de paix ou d'un traité de non prolifération nucléaire. Inutile ici en effet de tracer la différence de conséquences potentielles entre une position maximaliste dure dans une négociation de contrat entre Boeing ou Airbus et une compagnie aérienne et dans la renégociation de l'accord sur le nucléaire iranien. La présidence managériale magique et toute puissante (mais virtuelle) qui était celle de la campagne de Donald Trump n'est pas la présidence réelle de l'occupant de la Maison Blanche.

5 Par ailleurs, et toutes les données électorales le confirment, Donald Trump ne doit pas son élection à un parti qui l'a choisi, pour reprendre la formule d'un champion cycliste, «à l'insu de son plein gré ». Il peut donc se présenter comme libre de toute contrainte partisane. Mais les élus au Congrès du parti de l'éléphant ne doivent pas plus leur élection à Donald Trump dont la queue-de-pie électorale (coat tails en anglais) est quasiment inexistante. Dès le 9 novembre, une évidence paradoxale s'est imposée : Donald Trump a désormais terriblement besoin du Parti républicain (et le fait qu'il propose la nomination de Reince Priebus, le président du parti depuis janvier 2011 et donc pendant toute la durée de la campagne, comme secrétaire général de la Maison Blanche en est la première illustration patente), de la même manière que le Parti républicain, s'il veut atteindre les objectifs programmatiques qu'il s'est fixés dans les huit dernières années, a absolument besoin de Donald Trump. On notera que c'est la première fois depuis 1952 que les Républicains commencent le mandat de l'un des leurs à la Maison Blanche avec la majorité dans les deux chambres, 2001 n'ayant été qu'un moment très bref doublement interrompu par la défection du Sénateur Jeffords en mai et les attentats du 11 septembre.

6 Alors que l'on assiste depuis le début des primaires à un procès en illégitimité politique de l'establishment républicain de la part de Donald Trump, il lui faut maintenant se 
rendre à l'évidence : la conception et la conduite des politiques publiques ne peuvent pas être faites uniquement par d'anciens généraux et des grandes figures de Wall Street. Les élus républicains au Congrès, les gouverneurs, anciens ou en fonction, doivent être mobilisés, d'abord pour peupler le cabinet, ensuite pour établir un lien fonctionnel avec le Congrès qui détient les cordons de la bourse et reste maître des grands équilibres budgétaires, quand sa chambre haute ne se décharge pas d'une fonction constitutionnelle de la plus haute importance, la ratification des traités. C'est donc bien la majorité républicaine au Congrès, pas le président Trump, qui définira le montant de crédits alloués à une éventuelle politique de grands travaux qui heurte de plein front les nombreux tenants de l'équilibre budgétaire et de la réduction des dépenses publiques ; c'est aussi le Congrès qui aura le dernier mot sur l'avenir du Obamacare et donc de l'assurance maladie. En effet, même s'il existe chez les Républicains une quasi unanimité sur la volonté d'abroger la loi, se dégage aussi un consensus sur le fait d'abord qu'elle ne peut simplement rayée d'un trait de plume législatif puis présidentiel et qu'elle doit être remplacée par un autre système, ensuite que plusieurs des dispositions de cette loi sont aujourd'hui extrêmement populaires et devront donc être sauvées. Au lendemain de la mort de Fidel Castro et de l'ouverture d'une période de forte instabilité dans l'île, c'est le Congrès qui décidera s'il est opportun de maintenir l'embargo qui est, rappelons-le, une loi qui a été faite par les parlementaires américains en 1962 et que seuls ces derniers peuvent défaire.

7 Le Parti républicain devra aussi changer son logiciel idéologique car la forte demande d'État (en particulier d'État régalien) qui s'est exprimée chez des électeurs qui avaient un sentiment d'abandon ou de trahison devra se réconcilier avec la dénonciation d'un État liberticide, hyper-régulateur, envahissant et corrompu. C'est le syndrome traditionnel de la forteresse que l'on assiège puis que l'on prend et l'on désarme. De même, et de nombreux politistes l'ont souligné à l'instar de Matt Grossmann et David Hopkins dans leur remarquable, Asymmetric Politics, le Parti de l'éléphant est aujourd'hui enfermé dans une logique obstructionniste qui valorise la pureté idéologique conservatrice au détriment de toute forme de compromis. Il lui faut donc opérer un pivot opérationnel qui nécessite un changement radical de la culture institutionnelle du groupe républicain dans les deux chambres. Or ce changement doit s'opérer à l'intérieur d'un parti lui-même divisé et fracturé sur trois axes : entre sa base électorale et son leadership et son establishment, entre le président (qui est aussi le président du parti aux États-Unis) et le même establishment, entre différents courants qui composent une belle arborescence sur les sujets économiques, sociaux, ou de politique internationale.

Dernière chose, ces indispensables mutations institutionnelles et idéologiques devront se faire dans l'urgence pour deux raisons simples : d'abord, tous les présidents des États-Unis depuis Franklin Roosevelt sont jugés sur leurs premiers cent jours et quand bien même ces trois mois de février à avril 2017 sont une construction théorique et totalement artificielle, c'est néanmoins le filtre favori de la presse et des experts. Par ailleurs, Donald Trump a fait une campagne électorale qu'il a conçue comme une rencontre directe et sans filtre aucun avec les Américains. Tous les médiateurs traditionnels des campagnes ont été effacés, syndicats, partis, grands médias. Ils ne pourront pas, demain, être mobilisés pour servir d'amortisseur et d'isolation contre la déception d'un électorat dont les espoirs sont tellement importants et le sentiment de 
trahison et de colère contre les élites tellement fort. Donald Trump s'est imposé une obligation de résultats qui met potentiellement sa présidence en péril.

9 La présidence Trump était jusqu'au 8 novembre 2016 un récit (a narrative diraient les Américains). Il lui faut maintenant s'incarner. Raconter n'est pas décider.

\section{AUTEUR}

\section{VINCENT MICHELOT}

Vincent Michelot est professeur des universités à Sciences Po Lyon où il enseigne la politique américaine. Spécialiste des élections et des rapports entre les pouvoirs de l'État aux Etats-Unis, il est l'auteur de Kennedy (Paris, Gallimard, 2013). vincent.michelot@sciencespo-lyon.fr 